\title{
An individual difference perspective on focal versus nonfocal prospective memory
}

\author{
Sascha Zuber ${ }^{1}$ - Matthias Kliegel ${ }^{1,2} \cdot$ Andreas Ihle ${ }^{1,2,3}$
}

Published online: 28 June 2016

(C) Psychonomic Society, Inc. 2016

\begin{abstract}
The present study targeted the question of whether focal versus nonfocal prospective memory (PM) can be distinguished on a construct level, and if so, to what extent individual differences in these two constructs are related to individual differences in facets of controlled attention and episodic memory. 315 individuals (aged 20-68 years) were administered focal and nonfocal PM tasks as well as indicators measuring updating, inhibition, shifting, and episodic memory. Latent variable modeling revealed that focal and nonfocal PM were two distinguishable but related constructs. Furthermore, analyses showed that focal PM was more strongly related to inhibition, while nonfocal PM was more strongly related to shifting. Present data support the conceptual hypothesis that focal and nonfocal PM should be conceptualized as two distinguishable but related constructs. Moreover, they suggest that both have some but distinct associations to controlled attention.
\end{abstract}

Keywords Prospective remembering · Cue focality · Executive functions $\cdot$ Episodic memory $\cdot$ Latent variable modeling

Andreas Ihle

Andreas.Ihle@unige.ch

1 Department of Psychology, University of Geneva, Geneva, Switzerland

2 Center for the Interdisciplinary Study of Gerontology and Vulnerability, University of Geneva, Geneva, Switzerland

3 CIGEV, University of Geneva, Route des Acacias 54, 1227 Carouge, Switzerland
Prospective memory (PM) is defined as the processes involved in the formation and the delayed execution of intended actions (Kliegel, Mackinlay, \& Jäger, 2008a). PM plays a key role in many everyday tasks, as it enables us for example to remember to pay bills on time, to pick up children after school, or to call a friend on her birthday. PM has been conceptualized as a set of different process phases such as intention formation, intention retention, intention initiation and intention execution (Kliegel, Altgassen, Hering, \& Rose, 2011). In addition, PM tasks consist of both a prospective component (i.e., one needs to remember that a particular action has to be done as soon as the environmental conditions allow it; Einstein \& McDaniel, 1990, 1996) as well as a retrospective component (i.e., one needs to remember what that particular action was). In line with this conceptual view, empirical research suggests that retrospective memory (i.e., typically operationalized as episodic memory) may be a necessary, yet not sufficient processing resource for PM. For example, Burgess and Shallice (1997) showed that subjects with poor PM can achieve good episodic memory performance, but that subjects with poor episodic memory commonly tend to show also poor PM performance. Moreover, studies using latent variable modeling confirmed that PM and retrospective memory show both convergent and discriminant validity. Specifically, they found that individual differences in PM were to some extent related to individual differences in episodic memory, but also that a considerable amount of interindividual variance was not shared by the two constructs (correlations $r$ s ranging between .38 and .74; as reported by Salthouse, Berish, \& Siedlecki, 2004; Zeintl, Kliegel, \& Hofer, 2007). Thus, a certain association between episodic memory and PM has been established, possibly reflecting the process of storing the intention in episodic memory during the retention phase (Kliegel et al., 2011). However, from those data it is evident that other processes than episodic memory are additionally involved in PM. 
In theories of PM, those additional processes are commonly described by the prospective component of PM, comprising processes related to controlled attention, for example required to switch between the ongoing activity and the PM task (Kliegel et al., 2011). Conceptually, however, the consensus regarding the specific contribution of controlled attention to PM (and its task variants) remains under debate.

Different conceptual frameworks have attempted to specify the role of controlled attention in PM such as the multiprocess framework (McDaniel \& Einstein, 2000). According to this view, retrieval of a delayed intended action can be achieved through either strategic or automatic processes. Strategic processes that are assumed to demand controlled attention would be required when the individual has to actively monitor its environment for target cues that are related to the intention (e.g., visually "scanning" the street for a yellow post box in a shopping center to fulfill the intention of mailing a letter). In contrast, automatic processes that are assumed to demand less or no controlled attention would be supported by particularly salient cues in the environment that are assumed to automatically reactivate the intended action (e.g., seeing a large yellow post box in front of us that reminds us to mail the letter). According to the multiprocess framework, compared to individuals with fewer controlled attentional resources, individuals with more controlled attentional resources should perform better in PM tasks in which controlled attention is assumed to be required. In contrast, such an association between individual differences in controlled attention and individual differences in PM performance should be less pronounced for PM tasks that are assumed to rely on more automatic processing.

One of the PM task characteristics that have been conceptualized in the multiprocess framework to determine the demands for controlled attention is cue focality. A PM task is considered to be focal when the task-inherent processing of the stimuli for the ongoing task is overlapping with the processing required for PM cue detection (see also Einstein et al., 2005). For example, if the ongoing task was to tell whether a word presented at the bottom of a screen (e.g., apple) belongs to the category presented at the top of the screen (e.g., fruit), a focal PM task would be to remember to hit a particular button in response to a specific target word (e.g., peach). In this example, a nonfocal PM task would be to hit a particular button in response to a specific target string of letters (e.g., $e a c$ ), as the participant is processing words to categorize them, rather than treating them as letter strings. According to the multiprocess framework, focal and nonfocal PM tasks should differ in the particular processes they rely on. Specifically, nonfocal PM tasks are assumed to require more controlled attention than focal PM tasks.

Studies comparing these two PM task types largely confirmed the predictions of the multiprocess framework by distinguishing nonfocal from focal PM tasks for example in terms of different performance across those tasks in young versus older adults, adolescents versus young adults, mild cognitive impairment, dementia, or Parkinson's disease as well as on a neurochemical basis in e.g. nicotine deprived smokers (Costa et al., 2014; Henry, MacLeod, Phillips, \& Crawford, 2004; Ihle, Hering, Mahy, Bisiacchi, \& Kliegel, 2013; Kliegel, Phillips, \& Jäger, 2008b; McDaniel, Shelton, Breneiser, Moynan, \& Balota, 2011; Rusted, Trawley, Heath, Kettle, \& Walker, 2005; Scullin, McDaniel, Shelton, \& Lee, 2010; van den Berg, Kant, \& Postma, 2012; Wang et al., 2011). Therefore, these findings suggest that focal and nonfocal PM tasks measure (at least on some level) distinguishable types of PM that (partially) rely on different cognitive processes. However, to our knowledge there is no study so far that has comprehensively evaluated the degree of distinction between the two PM types on an associated process level. Therefore, the present study aimed to examine focal and nonfocal PM on a construct level in more detail. More precisely, as our first goal we aimed to analyze whether PM can be distinguished into these two constructs on a latent level, and if so, whether focal and nonfocal PM would be somehow related or whether they would represent entirely separable constructs.

As outlined, the multiprocess framework suggests that nonfocal PM tasks may demand high levels of controlled attentional resources, whereas focal PM tasks may rely more on automatic processes. Although there is general consensus that some PM tasks require more controlled attention than others, no study has so far examined the exact linkage of specific facets of controlled attention to both focal and nonfocal PM constructs in a single study. Early studies mostly used a global measure (rather than distinct facets of controlled attention) and found a significant association with PM (Insel, Morrow, Brewer, \& Figueredo, 2006; Martin, Kliegel, \& McDaniel, 2003; Salthouse et al., 2004). More recent studies then aimed to disentangle the particular role of the three facets of controlled attention, namely updating, inhibition, and shifting, as they were suggested by Miyake et al. (2000). In terms of task processing analysis, updating, for example, refers to the processes that are required to maintain and manipulate information that is processed, to continuously monitor its relevance, and to replace it with newer, more relevant information once it has become irrelevant for the ongoing task. Inhibition, for example, refers to the processes that are required to focus on a certain part of information available while suppressing other parts that are irrelevant or distracting as well as to suppress actions that are irrelevant or conflicting for the ongoing task. Shifting refers to the processes that are for example required to disengage from a particular strategy or ongoing task item and to engage in another cognitive set (e.g., that is related to the intention).

The few PM studies that have so far targeted the role of these specific facets of controlled attention in PM show some, although inconclusive, association between PM and controlled 
attention. Notably, all used either a focal or a nonfocal PM task and no study so far was designed to disentangle the processes related to the two PM task types. For example, inhibition, but neither shifting nor updating was found to be associated with focal PM (Kliegel, Ramuschkat, \& Martin, 2003; Mahy, Moses, \& Kliegel, 2014). Nonfocal PM performance, on the other hand, was found to be associated with inhibition and shifting, but not with updating (Schnitzspahn, Stahl, Zeintl, Kaller, \& Kliegel, 2013).

In summary, no conclusive pattern has been revealed so far that suggests a clear association between focal versus nonfocal PM and controlled attention. Some studies only used a single facet of controlled attention and others (that examined multiple facets of controlled attention) examined only one PM task type. To fill this gap, the second goal of the present study was to answer the question of whether focal and nonfocal PM share some but possibly not all of their underlying processes. Following the reasoning of a more general view of PM, focal and nonfocal PM should on one hand rely on similar processes. Specifically, in both PM task types, participants are required to form a particular intention, to retain that intention, to initiate it when the appropriate moment has come, and finally to execute the intention while being engaged in an ongoing task (Kliegel et al., 2011). On the other hand, focal and nonfocal PM should also be distinguishable because, according to the multiprocess framework (McDaniel \& Einstein, 2000), they differ in their demands on controlled attention, with focal PM tasks presumably being less demanding on controlled attention than nonfocal PM tasks. Thus, our goal was to focus on the potentially differential controlled attentional processes involved in focal and nonfocal PM tasks. In particular, we were interested to examine the associations of updating, inhibition, and shifting with PM, and whether these three facets of controlled attention show a differential pattern of associations with focal versus nonfocal PM.

Although clear a-priori predictions were difficult due to the lack of systematic comparisons of the two PM task types, based on the meta-analyses of Kliegel et al. (2008b) and Thle et al. (2013), we assumed that focal PM should also be related to some extent to controlled attention. Specifically, we predicted that focal PM performance should be related to inhibition. Participants performing a focal PM task should be "absorbed" by the ongoing task because they do not actively monitor the environment for a PM cue. This is because the focal PM target cue has to be processed in the same manner as the regular stimuli of the ongoing task and could be spontaneously retrieved (see McDaniel \& Einstein, 2000). Therefore, an appropriate PM response would first require to inhibit the dominant ongoing task response and then to execute the PM action as instructed (see for example Kliegel et al., 2003; Mahy et al., 2014, showing substantial associations of focal PM performance with inhibition). However, for nonfocal PM, different predictions seem reasonable. On the one hand, there may be a strong association of nonfocal PM with inhibition as reported by for example Schnitzspahn et al. (2013) if there is a need to inhibit the dominant ongoing task response before executing the PM action. On the other hand, in nonfocal PM tasks participants have to continuously shift between the completion of the ongoing task and to check for PM cues (e.g., Guynn, 2008; Schnitzspahn et al., 2013; see also the following paragraph). Therefore, the processing of the ongoing task may possibly be already continuously interrupted, which may reduce the need to refrain from the ongoing task response to the PM cue and lower the demand for inhibition.

Furthermore, for nonfocal PM we predicted that PM performance should be more strongly related to shifting. In nonfocal PM tasks, the defining features of the PM cues are not part of the information being processed during the ongoing task. Therefore, participants are required to actively monitor the environment to detect a PM cue. Consequently, a successful PM response would depend on the participant's capacity to periodically switch between completing the ongoing task and monitoring for the PM cue (see for example Schnitzspahn et al., 2013). Such shifting abilities may be best depicted by measuring specific switch costs, that is, the difference between mean response time (RT) in switch trials (i.e., those specific trials in which participants are required to switch to another type of mental processing) and mean RT in non-switch trials (i.e., trials with the same type of mental processing as in the preceding trial). This is because in PM the switch from performing a specific ongoing task trial to check for a PM cue (i.e., monitoring) may be triggered by that specific ongoing task trial. Therefore, the present study will focus on specific switch costs. As previous studies failed to find significant correlations between updating and either PM type, we did not have any particular hypothesis regarding the association between updating and PM, but rather aimed to explore a possibly differential association between updating and the two PM constructs.

As additional cognitive variable, we included episodic memory as a predictor. With no prior consensus on the association of episodic memory with focal/nonfocal PM (e.g., Ellis \& Kvavilashvili, 2000), we had no specific hypotheses, but aimed to account for potentially differential relations to focal versus nonfocal PM in our statistical models.

\section{Methods}

\section{Participants}

The sample consisted of 315 individuals aged from 20 to 68 years (continuous age sample; mean age $=41.5$ years, $S D=11.6$ ). 269 individuals $(85.4 \%)$ were women and $46(14.6 \%)$ were men. All participants gave informed consent and the present study included adherence to the declaration of Helsinki. 


\section{Materials}

Prospective memory We assessed PM in an event-based PM task with a focal and a nonfocal condition. In the ongoing task, participants were presented with a 2-back updating task, which has been successfully used in previous PM research in avoiding ceiling effects (West, Bowry, \& Krompinger, 2006). White uppercase letters (i.e., A, D, F, H, K, M, O, R, $\mathrm{T}$, and $\mathrm{Z}$ ), each surrounded by a differently colored frame (i.e., red, yellow, pink, light green, light blue, dark blue, dark green, turquoise, grey, and violet; order corresponds to the letters above) were serially displayed (in the same pseudorandomized order for all participants) in the center of a black background screen. Participants were asked to decide whether or not the present letter has occurred two stimuli ago by pressing either the right-arrow key for "Yes" or the left-arrow key for "No." The task started with a practice phase. Then, a first test block (in which no PM cue appeared, to assess baseline ongoing task performance) followed, for which 13 of the 50 stimuli were 2-back hit items. For the PM task that was embedded in the second and third test block, participants were instructed to remember to press the space bar whenever the letter was an "A" or a " $\mathrm{D}$ " (focal condition) or whenever the letter was surrounded by a red or a yellow frame (nonfocal condition) instead of undertaking the 2-back rating. Both PM conditions contained 50 stimuli of which 11 were 2-back hit items and four were PM cues. PM cues and 2-back hit items never occurred at the same time. For all blocks, after the appearance of a black screen with a white fixation cross for $1,000 \mathrm{~ms}$, each stimulus was presented for $2,000 \mathrm{~ms}$, followed by a black screen for $1,000 \mathrm{~ms}$. During this 3,000-ms time window for each stimulus, participants were asked to respond (i.e., pressing the ongoing task versus PM task related key). The two PM conditions were administered directly after each other with counterbalanced order of presentation, so that one half of the participants received the focal instruction first and the other half the nonfocal one. PM performance was the proportion of correct PM responses (i.e., pressing the space bar). Note that, as common in PM research, for PM performance, analyses were conducted only for individuals whose performance in the 2-back task was greater than $50 \%$ (excluding 27 individuals), thus assuring that they had to share resources between the ongoing and the PM task.

Updating We assessed updating in two different tests. First, performance in the first test block of the 2-back paradigm outlined above (in which no PM cue appeared) served as updating measure. The updating score was calculated as the number of 2-back hits subtracting false alarms in 2-back target trials.

Second, we adopted the keep-track task reported by Miyake et al. (2000). For this task, 33 German words, including four to six exemplars from each of six different categories (colors, countries, flowers, metals, sports, and relatives) served as stimuli. For each trial, a pseudo-randomized list of six to 15 words from four to five different categories was presented for 1,500 ms per word on a computer screen with the target categories remaining at the bottom of the screen. Participants were instructed to remember the last exemplar from each target category. At the end of each trial, these last exemplars had to be recalled, i.e. typed in to be directly recorded by the computer. As 2-3 exemplars from each target category were presented in each trial, a correct response required several instances of successful updating of working memory during a trial. To begin, participants performed a practice trial with two target categories. Then, they performed two test trials with three target categories and two with four target categories (i.e., not all of the presented categories were target categories of which participants needed to keep track, as typical in this task, see Miyake et al., 2000), with a total of 14 words to be recalled. The updating score was the total number of correctly recalled words across the four test trials.

Inhibition We assessed inhibition in two different tests. First, we used the Go/No-Go task (e.g., Newman, Widom, \& Nathan, 1985). In this task, stimuli consisted of a series of capital letters ranging from $\mathrm{A}$ to $\mathrm{Z}$ that were serially presented in the center of a computer screen with a duration varying between 750 and $1,250 \mathrm{~ms}$. Each trial began with a fixation cross appearing for 250-750 ms. The maximum trial length was $1,500 \mathrm{~ms}$. Participants were required to respond as fast as possible to any letter using the down-arrow key (Go trial), but not to respond when the letter " $\mathrm{X}$ " (No-Go trial) appeared on the screen. The No-Go rate was $20 \%$. The test block was presented for $3 \mathrm{~min}$, resulting in a varying trial number depending on individual's RT with a mean number of trials of $168.0(S D=9.4$, range: $148-207)$. The task started with a practice block of $30 \mathrm{~s}$.

Second, we used the stop-signal task (e.g., Logan, Schachar, \& Tannock, 1997). In this task, stimuli consisted of a sequence of black capital letters ranging from $\mathrm{A}$ to $\mathrm{Z}$ that were serially presented in the center of a computer screen for up to $1,000 \mathrm{~ms}$ and preceded by a fixation cross appearing for $500 \mathrm{~ms}$. The letter " $\mathrm{X}$ " was excluded, as it served as a No-Go stimulus in the Go/No-Go task outlined above. Participants were asked to discriminate vowels from consonants by using the left-arrow key (vowel) or the right-arrow key (consonant). Vowels and consonants were presented equally often. Further, participants were instructed not to respond whenever a letter changed from black to red during the trial (stop-signal). For those stop-trials, the delay between stimulus- and stop-signalappearance varied with stop-signal delays (SSDs) of 100, 200, and $300 \mathrm{~ms}$. The Stop-trial rate was $25 \%$. The test block was presented for $3 \mathrm{~min}$, resulting in a varying trial number depending on individual's RT with a mean number of trials of 
$139.6(\mathrm{SD}=9.5$, range: $120-174)$. The task started with a practice block of $60 \mathrm{~s}$.

For both inhibition tasks, we analyzed the proportion of correct rejections (i.e., inhibited responses in the No-Go/ stop-trials).

Shifting We assessed shifting in two different tests. In both tasks, as common in the literature (e.g., Miyake et al., 2000), participants were required to shift between two judgments during their processing of a set of bivalent stimuli (i.e., stimuli on which both judgments can be performed). First, we adopted a shifting task reported by Koch and Allport (2006), in the following called number-switch task. In this task, participants were presented with the digits 1-9 (excluding 5) in the center of a computer screen. Participants had to decide as fast as possible whether the digit was greater or less than 5 (magnitude judgment) or whether the digit was odd or even (parity judgment). For both judgments, responses were made by pressing either the left-arrow or the right-arrow key with the left or the right index finger, respectively. The type of judgment to perform was indicated by the respective question presented above the digit. The question and the digit remained on the screen until the response was made. Stimulus presentation order was random. Participants were told that their responses should be fast but accurate. They performed eight practice trials and after that 40 test trials for the magnitude judgment. Then, eight practice trials and 40 test trials followed for the parity judgment. Finally, there were 16 practice trials and 80 test trials in which both judgments had to be performed and type of judgment (magnitude versus parity) varied randomly from trial to trial. For all practice trials, participants received accuracy and RT feedback.

Second, we developed a parallel version of the numberswitch task called the perceptual-switch task where letters rather than digits were presented. Participants were presented with the letters A, B, d, e, F, H, r, and t in gray or red color. They had to decide as fast as possible whether it was an uppercase or a lowercase letter (capitalization judgment) or whether it was gray or red (color judgment). In all other respects, the perceptual-switch task was identical to the numberswitch task.

For both shifting tasks, responses with RTs greater than 4,000 or less than $100 \mathrm{~ms}$ were excluded $(0.6 \%$ of trials across participants). Specific switch costs were used as the dependent variable, computed as the difference between mean RT (in ms) in switch trials (i.e., trials in which participants were required to switch to the other type of judgment) and mean RT (in ms) in non-switch trials (i.e., trials with the same type of judgment as in the preceding trial) in the mixed-judgments block. The distribution of switching cost scores of all participants was reversed based on the sample mean so that higher values represented better performance across all variables. For each participant, participant's switching cost was subtracted from mean switching cost of the sample (i.e., mean-centering approach). Therefore, individuals with negative values had greater switch costs (and therefore lower shifting abilities) than the mean.

Episodic memory We assessed episodic memory in two different tests. First, we adopted the word pairs delayed subtest from the Wechler Memory Scale-Revised (WMS-R; Wechsler, 1987). Participants were presented with eight pairs of German words, of which four pairs were semantically related and four pairs were semantically unrelated. They had to memorize the pairings of the words. After giving an example, the experimenter read aloud all eight word pairs for $3 \mathrm{~s}$ each. Approximately $10 \mathrm{~min}$ later (following the number-switch task measuring shifting), the experimenter handed out the answer sheet which contained the first word of every word pair. Note that all eight first words were visible on the sheet but they were in a different order than they were memorized. Participants had $60 \mathrm{~s}$ in total to recall the second word of all eight word pairs. The episodic memory score was the total number of correctly completed word pairs $(\min =0 ; \max =8)$.

The second episodic memory task was delayed story recall (Härting et al., 2000). Participants were instructed that they would hear a short-story and asked to memorize as many details as possible because they would have to recall the story later during the testing session. Then, the experimenter read aloud the short-story and asked the participants again to not forget the story. Approximately $30 \mathrm{~min}$ after that, the experimenter handed out the (blank) answer sheet and asked the participants to recall the story by reporting as many details as possible. The episodic memory score was the total number of correctly reported details $(\min =0 ; \max =25)$.

\section{Procedure}

Participants were tested in groups of two to five. They were separated by partitions, which prevented them from seeing each other. The experimenter always assured that all in the group fully understood and followed the instructions. After a socio-demographic questionnaire, the cognitive tests were administered in the same pseudo-randomized order (i.e., PM, number-switch, word pairs delayed, Go/No-Go, perceptualswitch, stop-signal, delayed story recall, and keep-track) for all participants. Tasks measuring the same construct were intermixed with measures of other constructs and not administered directly one after another. The session lasted approximately one and a half hour and included two short breaks.

\section{Statistical analyses}

Latent variable modeling was conducted with the statistical computing software $R$, using the package lavaan (Rosseel, 2012). Latent variables for focal/nonfocal PM were based on 
deriving two indicators for each PM task type using a split-half parceling approach (DeVellis, 2012), i.e., averaged PM performance in PM target trials 1 and 2 for the first focal/nonfocal indicator as well as in PM target trials 3 and 4 for the second one. To evaluate how well the respective latent variable model (and thus the conceptual hypotheses) fitted the data, we evaluated the difference between the observed covariance matrix and the covariance matrix that was implied by the specified model. In detail, we used the following criteria to evaluate model fit: $\chi^{2}$ test (good models: $p$ value $>.10$ ), Comparative Fit Index (good models: $C F I>$.95), Incremental Fit Index (good models: $I F I>$ .95), Root Mean Square Error of Approximation (good models: RMSEA <.06), and Standardized Root Mean Square Residual (good models: $S R M R<.08$; see for example $\mathrm{Hu} \&$ Bentler, 1999 , for a discussion on recommended cutoff criteria for fit indexes in latent variable analyses). To evaluate the acceptability of a respective model over alternative models (and thus the acceptability of the conceptual hypotheses over alternative conceptual hypotheses), we statistically tested the change in model fit ( $\Delta \chi^{2}$ and its significance) by contrasting the respective models.

\section{Results}

\section{Descriptive statistics}

Table 1 shows means and standard deviations in the cognitive measures (i.e., observed variables). Table 2 displays bivariate correlations between the cognitive measures (i.e., observed variables) for descriptive purposes.

\section{Latent variable modeling}

The first goal of the present study was to target the questions whether focal and nonfocal PM can be distinguished on a construct level or whether they are better represented by a single, more general PM construct; and if the former, whether these two constructs are related or independent. To answer these questions, we contrasted several latent variable models by statistically testing change in model fit. Note that when referring to a "model" in the following sections, this refers to those statistical latent variable models, which then will have consequences for conceptual frameworks or theoretical views on PM. First, we applied a model in which the constructs of focal and nonfocal PM (with two indicators for each PM construct) were allowed to correlate freely (i.e., with the variances of both latent PM constructs being fixed to unity and with no other model parameters being constrained, allowing to estimate the factor loadings on both indicators of each latent PM construct; see Fig. 1). This model provided a good account of the data $\left(\chi^{2}\right.$ $=0.89, d f=1, p=.345, C F I>.99, I F I>.99$, RMSEA < .001, $S R M R=.01)$. In a next step, we contrasted this model with two
Table 1 Means and standard deviations of observed variables

\begin{tabular}{lll}
\hline Variable & $M$ & $S D$ \\
\hline 1. PM focal & .90 & .20 \\
2. PM nonfocal & .89 & .24 \\
3. 2-back & 4.39 & 6.28 \\
4. Keep-track & 8.73 & 2.45 \\
5. Go/No-Go & .80 & .14 \\
6. Stop-signal & .71 & .17 \\
7. Number-switch & 186.96 & 161.17 \\
8. Perceptual-switch & 105.82 & 114.07 \\
9. Word pairs delayed & 5.70 & 1.62 \\
10. Delayed story recall & 13.04 & 3.94 \\
\hline
\end{tabular}

alternative models: one in which the correlation between the constructs of focal and nonfocal PM was constrained to be equal to zero (i.e., besides that with no other model parameters being constrained) therefore representing two completely independent PM constructs and another alternative model in which the four PM indicators formed a single (unique) PM construct (i.e., with no separation into focal and nonfocal PM and with no other model parameters being constrained). Both alternative models provided a significantly worse model fit (two completely independent PM constructs: $\Delta \chi^{2}=42.24, \Delta d f=1, p<.001$; single unique PM construct: $\Delta \chi^{2}=30.87, \Delta d f=1, p<.001$ ). Thus, these results endorse as the most appropriate model that in which focal and nonfocal PM were represented by separate but related constructs.

Latent constructs of focal and nonfocal PM being established, our second study goal was to focus on the potentially differential controlled attentional and episodic memory processes involved in focal and nonfocal PM. In particular, we were interested to examine the associations of updating, inhibition, and shifting as well as episodic memory with focal and nonfocal PM, and whether the three facets of controlled attention and episodic memory show a different pattern of associations with focal versus nonfocal PM. For this purpose, we added the three facets of controlled attention as well as episodic memory as latent constructs to the model in which focal and nonfocal PM were represented by separate but related constructs (see Fig. 2). This model also provided a good account of the data $\left(\chi^{2}=36.69\right.$, $d f=39, p=.576, C F I>.99$, IFI $>.99$, RMSEA $<.001$, SRMR $=$ $.03)$. In this model, updating and inhibition were significantly correlated only with focal (but not with nonfocal) PM (see also Table 3 for an overview regarding the correlations between all latent variables, as implied by the specified model). Shifting was significantly correlated only with nonfocal (but not with focal) PM. Episodic memory was significantly correlated with the two PM constructs.

To test whether the three facets of controlled attention and episodic memory show a different pattern of associations with focal versus nonfocal PM, we subsequently contrasted this 
Table 2 Correlations between observed variables

\begin{tabular}{|c|c|c|c|c|c|c|c|c|c|}
\hline Variable & 1 & 2 & 3 & 4 & 5 & 6 & 7 & 8 & 9 \\
\hline 1. PM focal & - & & & & & & & & \\
\hline 2. PM nonfocal & $.36 * * *$ & - & & & & & & & \\
\hline 3. 2-back & $.19 * *$ & $.17 * *$ & - & & & & & & \\
\hline 4. Keep-track & $.12 *$ & $.12 *$ & $.33 * * *$ & - & & & & & \\
\hline 5. Go/No-Go & $.19 * *$ & $.12 *$ & $.00 \mathrm{~ns}$ & $.00 \mathrm{~ns}$ & - & & & & \\
\hline 6. Stop-signal & $.12 *$ & $.07 \mathrm{~ns}$ & $-.07 \mathrm{~ns}$ & $.03 \mathrm{~ns}$ & $.36 * * *$ & - & & & \\
\hline 7. Number-switch & $.06 \mathrm{~ns}$ & $.12 *$ & $.12 *$ & $.05 \mathrm{~ns}$ & $.12 *$ & $.06 \mathrm{~ns}$ & - & & \\
\hline 8. Perceptual-switch & $.08 \mathrm{~ns}$ & $.12 *$ & $-.04 \mathrm{~ns}$ & $-.02 \mathrm{~ns}$ & $.13^{*}$ & $.00 \mathrm{~ns}$ & $.35 * * *$ & - & \\
\hline 9. Word pairs delayed & $.22 * * *$ & $.15^{*}$ & $.26 * * *$ & $.31 * * *$ & $.15^{* *}$ & $.16^{* *}$ & $.12 *$ & $.04 \mathrm{~ns}$ & - \\
\hline 10. Delayed story recall & $.16 * *$ & $.12 *$ & $.25 * * *$ & $.31 * * *$ & $.11^{\prime}$ & $.02 \mathrm{~ns}$ & $.09 \mathrm{~ns}$ & $.06 \mathrm{~ns}$ & $.43 * * *$ \\
\hline
\end{tabular}

Note. Correlation matrix regarding the relations between observed variables. Higher values represented better performance across all variables $* * * p<.001 ; * * p<.01 ; * p<.05 ; ’ p<.10$, trend-wise significant; ns = non-significant, $p>.10$

model with four alternative models: Constraining the correlation of inhibition with the two PM constructs to be equal for focal and nonfocal PM (versus when they are allowed to vary) provided a significantly worse model fit $\left(\Delta \chi^{2}=5.43, \Delta d f=1, p=\right.$ .020). Together with the aforementioned pattern of correlations between the latent constructs, this indicated that the relation of inhibition with focal PM was substantially larger than with nonfocal PM. Likewise, constraining the correlation of shifting with the two PM constructs to be equal for focal and nonfocal PM (versus when they are allowed to vary) provided a significantly worse model fit $\left(\Delta \chi^{2}=5.10, \Delta d f=1, p=.024\right)$. Together with the aforementioned pattern of correlations between the latent constructs, this indicated that the relation of shifting with nonfocal PM was substantially larger than with focal PM. However, constraining the correlations of updating or episodic memory with the two PM constructs to be equal for focal and nonfocal PM (versus when they are allowed to vary) provided no significant change in model fit (updating: $\Delta \chi^{2}=0.81, \Delta d f=$ $1, p=.370$; episodic memory: $\Delta \chi^{2}=1.54, \Delta d f=1, p=.215$ ). This indicated that the relation of updating/episodic memory with focal PM did not differ in size compared to nonfocal PM. Moreover, as in the previous set of analyses, setting the correlation between the constructs of focal and nonfocal PM equal to zero or applying a unique PM construct provided a significantly worse model fit (two completely independent PM constructs:

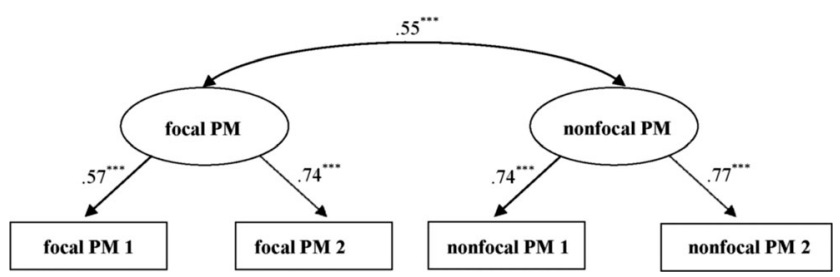

Fig. 1 Latent variable model of focal and nonfocal prospective memory $(\mathrm{PM})$ as distinct but freely correlating constructs
$\Delta \chi^{2}=101.48, \Delta d f=1, p<.001$; single unique PM construct: $\left.\Delta \chi^{2}=145.86, \Delta d f=5, p<.001\right)^{1}$

\section{Discussion}

Our first study goal was to evaluate the distinguishability of focal and nonfocal PM on a construct level. To do so, we contrasted three different statistical models. In our hypothesized model, we depicted focal and nonfocal PM as two distinct constructs that were allowed to correlate freely. This model should have the best fit if PM is indeed discriminable into two different constructs that share some but not all of their underlying processes. The correlation among the two latent variables would indicate to what degree focal and nonfocal PM share common processes. In a first alternative model, PM was depicted as a single (unique) construct. In a second alternative model, the correlation between focal and nonfocal PM was constrained to zero, therefore representing two completely independent PM constructs. A set of analyses based on latent

\footnotetext{
${ }^{1}$ We additionally repeated all analyses with the following alternative measures: latent variables for focal/nonfocal PM based on an odd-even parceling approach (i.e., averaged PM performance in trials 1 and 3 for the first focal/nonfocal indicator as well as in trials 2 and 4 for the second one); the sensitivity index $d^{\prime}$ as updating score in the 2-back task; a measure that takes the individual's response latency in the Go/No-Go/ stop-signal task into account (i.e., by dividing the respective inhibition score by the individual's mean RT in correct Go-trials) to control for the possibility that a good inhibition performance was achieved by a deliberately slowed down processing to avoid mistakes; and a measure that takes the individual's response latency in the number-switch/perceptual-switch task into account (i.e., by dividing the respective shifting score by the individual's mean RT in non-switch trials) to derive a proportion score that expresses the magnitude of an individual's switching cost as a percentage of his/her average response latency (cf. Schnitzspahn et al. 2013). These additional analyses revealed the same pattern of results.
} 


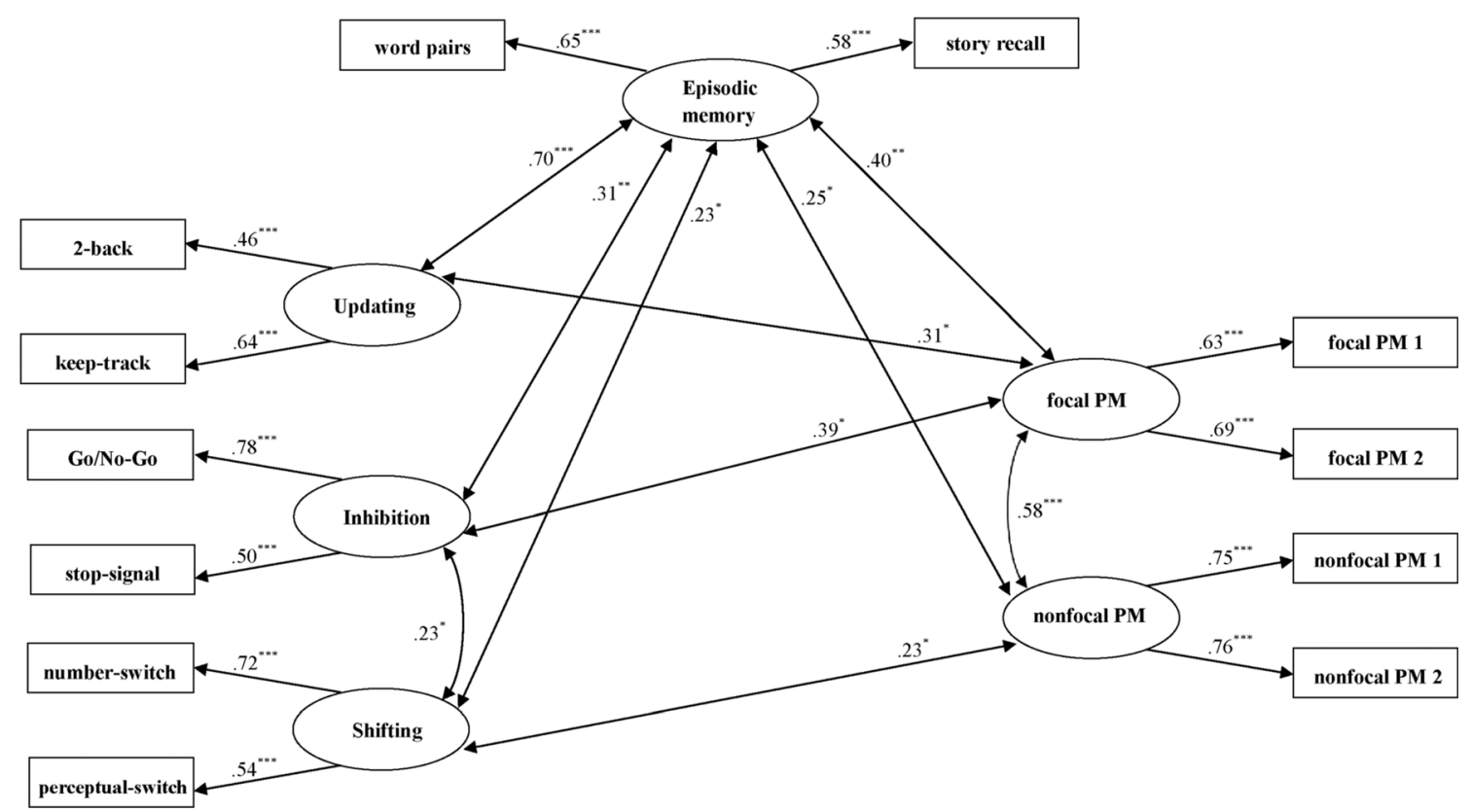

Fig. 2 Latent variable model of focal and nonfocal prospective memory (PM) as distinct but freely correlating constructs and their relations to the facets of controlled attention and episodic memory (with only significant correlations displayed)

variable modeling revealed that the hypothesized model indeed provided a significantly better model fit to the data (compared to the two alternative models), empirically confirming for the first time that focal and nonfocal PM represent two identifiable, distinct but related constructs. This has some important conceptual implications for current theoretical views on PM.

The distinguishability of focal and nonfocal PM we observed is in line with key assumptions of the multiprocess framework (McDaniel \& Einstein, 2000) and with several studies that found different performance for focal and nonfocal PM tasks (e.g., Cherry et al., 2001; Einstein et al., 2005; Einstein, Richardson, Guynn, Cunfer, \& McDaniel, 1995, Experiment 2; Kvavilashvili, Kornbrot, Mash, Cockburn, \& Milne, 2009). Furthermore, studies comparing PM task performance in groups of younger versus older adults also indicated the distinguishability of focal and nonfocal PM. For example, Vogels, Dekker, Brouwer, and de Jong (2002) found younger and older adults performing comparably well in nonfocal, but not in focal, PM tasks. Rendell, McDaniel, Forbes, and Einstein (2007) in return found performance in focal PM tasks to be better only in older but not in younger adults. Furthermore, Niedzwienska and Barzykowski (2012) found PM performance to depend on cue focality in young, middle-aged, and older adults. Although most of the previous literature has proposed focal and nonfocal PM tasks to be distinct concepts, the present study finally allows for the first time to confirm focal and nonfocal PM being conceptualized as distinct but related constructs.

As complementary second goal, we aimed to focus on the potentially differential processes that contribute to individual differences in focal and nonfocal PM tasks. Specifically, we aimed at disentangling the association patterns of the three facets of controlled attention updating, inhibition, and shifting as well as episodic memory with focal and nonfocal PM. Latent variable modeling indicated that inhibition and shifting were differently associated with focal and nonfocal PM. In

Table 3 Correlations between latent variables

\begin{tabular}{|c|c|c|c|c|c|}
\hline Construct & 1 & 2 & 3 & 4 & 5 \\
\hline 1. PM focal & - & & & & \\
\hline 2. PM nonfocal & $.58(<.001)$ & - & & & \\
\hline 3. Updating & $.31(.024)$ & $.20(.115)$ & - & & \\
\hline 4. Inhibition & $.39(.021)$ & $.19(.164)$ & $.10(.462)$ & - & \\
\hline 5. Shifting & $.04(.692)$ & $.23(.033)$ & $.12(.498)$ & $.23(.019)$ & - \\
\hline 6. Episodic memory & $.40(.001)$ & $.25(.028)$ & $.70(<.001)$ & $.31(.008)$ & $.23(.035)$ \\
\hline
\end{tabular}

Note. Correlation matrix regarding the relations between latent variables. Higher values represent better performance across all variables. Values in parentheses are $p$ values 
detail, inhibition was found to be significantly related to focal PM performance, but not to nonfocal PM performance. In addition, tests of change in model fit (together with this pattern of correlations between the latent constructs) indicated that the relation of inhibition with focal PM was reliably larger than with nonfocal PM. Conceptually, this can be seen as being in line with predictions from the multiprocess framework (McDaniel \& Einstein, 2000): During a focal PM task, participants are likely more absorbed in the ongoing task as they are not required to continuously monitor the environment for PM cues, as the cues would "pop out" of the regular stimuli while performing the ongoing task and the intention could be spontaneously retrieved. Therefore, when encountering the PM target cue, participants have to inhibit the dominant ongoing task response to successfully perform the PM action (see also e.g. Kliegel et al., 2003; Mahy et al., 2014, showing substantial associations of focal PM performance with inhibition capabilities).

Notably, we did not find a substantial association of inhibition with nonfocal PM as reported by for example Schnitzspahn et al. (2013). In nonfocal PM tasks, participants are required to continuously monitor for PM cues (Guynn, 2008; McDaniel \& Einstein, 2000). Specifically, participants have to continuously shift between completing the ongoing task and checking for PM cues. Therefore, the processing of the ongoing task is already continuously interrupted, which may reduce the need to refrain from the ongoing task response to the PM cue and lower the demand for inhibition.

Moreover, as postulated and again in accordance with the multiprocess framework (McDaniel \& Einstein, 2000), shifting was significantly correlated with nonfocal PM performance, but not with focal PM performance. In addition, tests of change in model fit (together with this pattern of correlations between the latent constructs) indicated that the relation of shifting with nonfocal PM was reliably larger than with focal PM. This corroborates the view that in nonfocal PM tasks, participants continuously have to switch between the completion of the ongoing task and the monitoring of the environment for a PM cue (see also Guynn, 2008; Schnitzspahn et al., 2013). Furthermore, analyses showed that this association of shifting with PM performance did not emerge in focal PM. This is in line with the assumption that, in focal PM tasks, participants may offload processes required in nonfocal tasks for cue monitoring and cue detection to the environment (allowing spontaneous retrieval of the intention; see McDaniel \& Einstein, 2000). Therefore, the need to continuously switch between cue monitoring and the processing of the ongoing task is substantially decreased.

The assessment of the association of updating to the two PM constructs was more exploratory. Our data showed a significant correlation between updating and focal (but not nonfocal) PM. However, tests of change in model fit indicated that the relation of updating with focal PM did not differ in size compared to nonfocal PM (note that, descriptively, the correlation for nonfocal PM was about the same size as for focal PM and slightly missed the .10 significance level). Thus, at least for focal $\mathrm{PM}$, the observed correlation with updating is in some contradiction with previous studies that did not find a substantial relation between updating and either focal or nonfocal PM. A correlation between updating and PM in the present study, however, is likely due to the fact that we used an updating paradigm as ongoing task. Thus, participants with more updating capacities are likely to perform the ongoing 2-back task easier, which in consequence would free more resources that could be allocated to the PM task if necessary. Hence, future research will have to elaborate on the association pattern we obtained, using a variety of ongoing tasks in the two PM paradigms.

A further target of the present study was the relation of episodic memory with PM. With no prior consensus on a possibly differential association of episodic memory with focal versus nonfocal PM, we did not formulate specific hypotheses regarding the association of episodic memory with the two PM constructs. Our data analysis shows that, on a construct level, episodic memory is significantly related to both focal and nonfocal PM. This is in accordance with other studies that found PM to be a construct distinct from but to some extent related to episodic memory, hence with both discriminant and convergent validity (Salthouse et al., 2004; Zeintl et al., 2007). Notably, on a descriptive level, the relation of episodic memory to PM was remarkably large in focal PM. However, tests of change in model fit indicated that the relation of episodic memory with focal PM did not differ in size compared to nonfocal PM. Conceptually, in focal PM tasks, participants can rely on more spontaneous retrieval (McDaniel \& Einstein, 2000) and do not necessarily have to continuously monitor for PM cues. Therefore, to keep the intention available (at least on a minimum level), episodic memory processes are required (see the conceptual view on the retrospective component in PM, Einstein \& McDaniel, 1990, 1996). Present results suggest that episodic memory also seems to be a key capacity in nonfocal PM. However, future research will have to study different PM paradigms to clarify whether episodic memory is a universal component of PM (as the present results suggest) or whether certain conditions decrease the load on episodic long-term memory required for successful prospective remembering.

Conceptually important, the present results may help to shape extant theories of the processes contributing to focal versus nonfocal PM. Specifically, the contribution of controlled attention to focal PM may not be anticipated by some conceptualizations of the distinction between focal versus nonfocal PM, where it has been suggested that in focal PM tasks "the cue is sufficiently processed to enable involuntary (automatic) retrieval of the intended action" (McDaniel \& Einstein, 2000, p. 136). At least present data do not support such strong positions of no contribution of controlled attention to focal PM. However, present results are in line with recent meta-analyses on the role of cue 
focality, which revealed that PM age effects were present even for focal PM tasks, suggesting that focal PM may also be related to some extent to controlled attention (Ihle et al., 2013; Kliegel et al., 2008b). This underlines the need to extend PM models from retrieval processes (such as PM cue detection and retrieval of the intention) to post-retrieval processes (such as response management and intention execution) and to specify their particular demands on controlled attention. An example may be inhibitory capacities possibly required for response management to execute the intended action in the post-retrieval phase (e.g., Ihle et al., 2013): Given that in focal PM the intention may be relatively automatically retrieved, an appropriate execution of the PM action in focal PM tasks would first require to inhibit the dominant ongoing task response and then to carry out the PM action (see for example Ihle et al., 2013; Kliegel et al., 2003; Mahy et al., 2014). Therefore, an appropriate conceptualization of focal versus nonfocal PM may not be a distinction between automatic or spontaneous versus controlled processing of PM, but rather a more fine-grained perspective that considers the coexisting contribution of automatic and controlled processes within the different process phases of PM. This may also help to explain the mixed results across studies comparing focal versus nonfocal PM performance (e.g., Loft \& Remington, 2013; Rendell et al., 2007; Vogels et al., 2002).

Limitations of the present study concern the number of indicators for the latent constructs. We acknowledge that it may be more desirable to model latent variables based on three different tasks per ability. However, it is also an acceptable approach to use only two tasks per ability: Specifically, while three indicators are necessary in order to be able to estimate models with a single latent construct, for models with more than one latent construct, at least two indicators are necessary for each latent construct (Kline, 2010; König, Messmann, Mulder, \& De Maeyer, 2015). Likewise, it would be desirable to model each of the latent PM variables based on (at least) two separate tasks. However, it may be also an acceptable approach to derive two indicators for a latent variable based on a single task using a parceling approach (e.g., split half), as in the present study. The reasoning behind such parceling approaches (as common also in for example scale development) is a natural extension of the parallel-tests model (DeVellis, 2012). Therefore, each test item can be regarded as a parallel test measuring the same construct. Hence, one can also regard the two halves of a set of test items as parallel tests measuring the same construct. With the applied split-half parceling approach, we modeled this conceptual reasoning that allowed eliminating variance in each test not being influenced by the latent variable, since each test half can be regarded as a parallel (not identical) test (DeVellis, 2012). However, future research will have to replicate the observed pattern of results by modeling three tasks per ability and using different PM paradigms for each PM task type.

The order of task administration is a challenge for research that focuses on classic experimental but also on individual difference research questions, as in the present study. We anticipated practice effects across PM tasks and therefore counterbalanced administration of the two PM conditions to control for training effect-related variance in the second administered condition. We acknowledge that when examining individual differences, counterbalancing the administration of tasks may lead to a subject $x$ treatment interaction in some cases. However, the order of focal/nonfocal PM task administration in the present study did not moderate the observed pattern of results ( $p s>.158$ ).

In sum, the current study allowed for the first time to statistically model focal and nonfocal PM as two distinct but related constructs that have convergent and discriminant validity. Furthermore, results facilitate a clearer understanding of the association between different facets of controlled attention and PM, by dismantling the associations between the facets with focal and nonfocal PM. Specifically, latent variable modeling revealed a distinctive association pattern between controlled attention and PM, with focal PM being more strongly related to inhibition capacities and with nonfocal PM being more strongly related to shifting processes. Therefore, the present study may help to further elucidate the (differential) controlled attentional processes in focal and nonfocal PM that have been discussed in the literature for more than two decades (Einstein \& McDaniel, 1990; Ihle et al., 2013; Kliegel et al., 2008b; McDaniel \& Einstein, 2000).

Acknowledgments The authors declare no conflicts of interest.

Matthias Kliegel acknowledges support from the Swiss National Science Foundation (SNSF).

Matthias Kliegel and Andreas Ihle belong to the Swiss National Centre of Competences in Research LIVES-Overcoming vulnerability: life course perspectives.

\section{References}

Burgess, P. W., \& Shallice, T. (1997). The relationship between prospective and retrospective memory: Neuropsychological evidence. In M. A. Conway (Ed.), Cognitive models of memory (pp. 247-272). UK: MIT Press.

Cherry, K. E., Martin, R. C., Simmons-D’Gerolamo, S. S., Pinkston, J. B., Griffing, A., \& Gouvier, D. (2001). Prospective remembering in younger and older adults: Role of the prospective cue. Memory, 9(3), 177-193.

Costa, A., Peppe, A., Serafini, F., Zabberoni, S., Barban, F., Caltagirone, C., \& Carlesimo, G. A. (2014). Prospective memory performance of patients with Parkinson's disease depends on shifting aptitude: evidence from cognitive rehabilitation. Journal of the International Neuropsychological Society, 20(7), 717-726. doi:10.1017/s1355617714000563

DeVellis, R. F. (2012). Scale development: Theory and applications. Los Angeles: SAGE Publications.

Einstein, G. O., \& McDaniel, M. A. (1990). Normal aging and prospective memory. Journal of Experimental Psychology: Learning, Memory, and Cognition, 16(4), 717-726. doi:10.1037/0278-7393.16.4.717

Einstein, G. O., \& McDaniel, M. A. (1996). Retrieval processes in prospective memory: Theoretical approaches and some new empirical findings. In M. Brandimonte, G. O. Einstein, \& M. A. McDaniel (Eds.), Prospective memory: Theory and applications. Mahwah: Lawrence Erlbaum Associates. 
Einstein, G. O., McDaniel, M. A., Thomas, R., Mayfield, S., Shank, H., Morrisette, N., \& Breneiser, J. (2005). Multiple processes in prospective memory retrieval: Factors determining monitoring versus spontaneous retrieval. Journal of Experimental Psychology: General, 134(3), 327342. doi:10.1037/0096-3445.134.3.327

Einstein, G. O., Richardson, S. L., Guynn, M. J., Cunfer, A. R., \& McDaniel, M. A. (1995). Aging and prospective memory - examining the influences of self-initiated retrieval-processes. Journal of Experimental Psychology: Learning, Memory, and Cognition, 21(4), 996-1007. doi:10.1037/0278-7393.21.4.996

Ellis, J., \& Kvavilashvili, L. (2000). Prospective memory in 2000: Past, present, and future directions. Applied Cognitive Psychology, 14, 1-9. doi:10.1002/Acp.767.Abs

Guynn, M. J. (2008). Theory of monitoring in prospective memory: Instantiating a retrieval mode and periodic target checking. In M. Kliegel, M. A. McDaniel, \& G. O. Einstein (Eds.), Prospective memory: Cognitive, neuroscience, developmental, and applied perspectives (pp. 161-185). London: Lawrence Erlbaum.

Härting, C., Markowitsch, H. J., Neufeld, H., Calabrese, R., Deisinger, K., \& Kessler, J. (2000). Wechsler gedächtnistest - revidierte fassung (WMS-R). Bern: Huber.

Henry, J. D., MacLeod, M. S., Phillips, L. H., \& Crawford, J. R. (2004). A meta-analytic review of prospective memory and aging. Psychology and Aging, 19(1), 27-39. doi:10.1037/0882-7974.19.1.27

Hu, L., \& Bentler, P. M. (1999). Cutoff criteria for fit indexes in covariance structure analysis: Conventional criteria versus new alternatives. Structural Equation Modeling, 6, 1-55.

Ihle, A., Hering, A., Mahy, C. E. V., Bisiacchi, P. S., \& Kliegel, M. (2013). Adult Age differences, response management, and Cue focality in event-based prospective memory: A meta-analysis on the role of task order specificity. Psychology and Aging, 28(3), 714-720. doi:10.1037/A0033653

Insel, K. C., Morrow, D., Brewer, B., \& Figueredo, A. (2006). Executive function, working memory, and medication adherence among older adults. The Journals of Gerontology Series B: Psychological Sciences and Social Sciences, 61(2), 102-107.

Kliegel, M., Altgassen, M., Hering, A., \& Rose, N. S. (2011). A processmodel based approach to prospective memory impairment in Parkinson's disease. Neuropsychologia, 49(8), 2166-2177. doi:10.1016/J.Neuropsychologia.2011.01.024

Kliegel, M., Mackinlay, R., \& Jäger, T. (2008). A life-span approach to the development of complex prospective memory. In M. Kliegel, M. A. McDaniel, \& G. O. Einstein (Eds.), Prospective memory: Cognitive, neuroscience, developmental, and applied perspectives (pp. 187216). Mahwah: Lawrence Erlbaum Associates.

Kliegel, M., Phillips, L. H., \& Jäger, T. (2008). Adult age differences in event-based prospective memory: A meta-analysis on the role of focal versus nonfocal cues. Psychology and Aging, 23(1), 203-208. doi:10.1037/0882-7974.23.1.203

Kliegel, M., Ramuschkat, G., \& Martin, M. (2003). Executive functions and prospective memory performance in old age: An analysis of event-based and time-based prospective memory. Zeitschrift Für Gerontologie Und Geriatrie, 36(1), 35-41. doi:10.1007/S00391-003-0081-5

Kline, R. B. (2010). Principles and practice of structural equation modeling (3rd ed.). London: Guilford Press.

Koch, I., \& Allport, A. (2006). Cue-based preparation and stimulus-based priming of tasks in task switching. Memory \& Cognition, 34(2), 433444. doi:10.3758/Bf03193420

König, C., Messmann, G., Mulder, R. H., \& De Maeyer, S. (2015). Accounting for complexity: Structural equation modeling (SEM) in HRD research. In M. N. K. Saunders \& P. Tosey (Eds.), Handbook of research methods on human resource development (pp. 273-290). Northampton: Edward Elgar Publishing, Incorporated.

Kvavilashvili, L., Kornbrot, D. E., Mash, V., Cockburn, J., \& Milne, A. (2009). Differential effects of age on prospective and retrospective memory tasks in young, young-old, and old-old adults. Memory, 17(2), 180-196. doi:10.1080/09658210802194366
Loft, S., \& Remington, R. W. (2013). Wait a second: Brief delays in responding reduce focality effects in event-based prospective memory. Quarterly Journal of Experimental Psychology, 66(7), 1432-1447.

Logan, G. D., Schachar, R. J., \& Tannock, R. (1997). Impulsivity and inhibitory control. Psychological Science, 8(1), 60-64. doi:10.1111 j.1467-9280.1997.tb00545.x

Mahy, C. E. V., Moses, L. J., \& Kliegel, M. (2014). The impact of age, ongoing task difficulty, and cue salience on preschoolers' prospective memory performance: The role of executive function. Journal of Experimental Child Psychology, 127, 52-64. doi:10.1016/j. jecp.2014.01.006

Martin, M., Kliegel, M., \& McDaniel, M. A. (2003). The involvement of executive functions in prospective memory performance of adults. International Journal of Psychology, 38(4), 195-206. doi:10.1080 /00207590244000205

McDaniel, M. A., \& Einstein, G. O. (2000). Strategic and automatic processes in prospective memory retrieval: A multiprocess framework. Applied Cognitive Psychology, 14, 127-144. doi:10.1002/Acp.775

McDaniel, M. A., Shelton, J. T., Breneiser, J. E., Moynan, S., \& Balota, D. A. (2011). Focal and nonfocal prospective memory performance in very mild dementia: A signature decline. Neuropsychology, 25(3), 387-396. doi:10.1037/A0021682

Miyake, A., Friedman, N. P., Emerson, M. J., Witzki, A. H., Howerter, A., $\&$ Wager, T. D. (2000). The unity and diversity of executive functions and their contributions to complex "frontal lobe" tasks: A latent variable analysis. Cognitive Psychology, 41(1), 49-100. doi:10.1006 /cogp.1999.0734

Newman, J. P., Widom, C. S., \& Nathan, S. (1985). Passive-avoidance in syndromes of disinhibition - psychopathy and extraversion. Journal of Personality and Social Psychology, 48(5), 1316-1327. doi:10.1037 /0022-3514.48.5.1316

Niedzwienska, A., \& Barzykowski, K. (2012). The age prospective memory paradox within the same sample in time-based and event-based tasks. Aging, Neuropsychology, and Cognition, 19(1-2), 58-83. doi:10.1080/13825585.2011.628374

Rendell, P. G., McDaniel, M. A., Forbes, R. D., \& Einstein, G. O. (2007). Age-related effects in prospective memory are modulated by ongoing task complexity and relation to target cue. Aging, Neuropsychology, and Cognition, 14(3), 236-256. doi:10.1080/13825580600579186

Rosseel, Y. (2012). Lavaan: An R package for structural equation modeling. Journal of Statistical Software, 48(2), 1-36.

Rusted, J. M., Trawley, S., Heath, J., Kettle, G., \& Walker, H. (2005). Nicotine improves memory for delayed intentions. Psychopharmacology, 182(3), 355-365. doi:10.1007/s00213-005-0109-1

Salthouse, T. A., Berish, D. E., \& Siedlecki, K. L. (2004). Construct validity and age sensitivity of prospective memory. Memory \& Cognition, 32(7), 1133-1148. doi:10.3758/Bf03196887

Schnitzspahn, K. M., Stahl, C., Zeintl, M., Kaller, C. P., \& Kliegel, M. (2013). The role of shifting, updating, and inhibition in prospective memory performance in young and older adults. Developmental Psychology, 49(8), 1544-1553. doi:10.1037/a0030579

Scullin, M. K., McDaniel, M. A., Shelton, J. T., \& Lee, J. H. (2010). Focal/ nonfocal Cue effects in prospective memory: Monitoring difficulty or different retrieval processes? Journal of Experimental Psychology: Learning, Memory, and Cognition, 36(3), 736-749. doi:10.1037 /A0018971

van den Berg, E., Kant, N., \& Postma, A. (2012). Remember to buy milk on the Way home! a meta-analytic review of prospective memory in mild cognitive impairment and dementia. Journal of the International Neuropsychological Society, 18(4), 706-716. doi:10.1017 /S1355617712000331

Vogels, W. W. A., Dekker, M. R., Brouwer, W. H., \& de Jong, R. (2002). Age-related changes in event-related prospective memory performance: A comparison of four prospective memory tasks. Brain and Cognition, 49(3), 341-362. doi:10.1006/brcg.2001.1504 
Wang, L. J., Altgassen, M., Liu, W., Xiong, W. R., Akgün, C., \& Kliegel, M. (2011). Prospective memory across adolescence: The effects of age and cue focality. Developmental Psychology, 47(1), 226-232. doi:10.1037/a0021306

Wechsler, D. (1987). Wechsler memory scale, revised manual. San Antonio: Psychological Corp.

West, R., Bowry, R., \& Krompinger, J. (2006). The effects of working memory demands on the neural correlates of prospective memory. Neuropsychologia, 44(2), 197-207. doi:10.1016/J. Neuropsychologia.2005.05.003

Zeintl, M., Kliegel, M., \& Hofer, S. A. (2007). The role, of processing resources in age-related prospective and retrospective memory within old age. Psychology and Aging, 22(4), 826-834. doi:10.1037/08827974.22.4.826 